\title{
COVID self-certification: recognition of postgraduate training and experiences gained during the pandemic
}

\author{
Authors: Patience Ehilawa, ${ }^{\mathrm{A}}$ Fiona Thompson, ${ }^{\mathrm{B}}$ Sarah Muldoon, ${ }^{\mathrm{C}}$ Rana Ahmed, ${ }^{\mathrm{A}}$ Mary Ariyo, ${ }^{\mathrm{A}}$ Jonathan Corne ${ }^{\mathrm{D}}$ \\ and Paul Sadler ${ }^{\mathrm{E}}$
}

\section{Introduction}

The COVID-19 pandemic has placed significant pressures on healthcare services and professionals delivering patient care. Consequently, postgraduate medical training has been interrupted, with limited opportunities for supervised workplace-based assessments. Therefore, additional knowledge and skills gained, particularly those outside the scope of respective training curriculum, may not be robustly captured. This project aimed to identify trainees' views on additional capabilities gained during the pandemic and the value of self-certification, in order to inform the design of a COVID self-certification template.

\section{Methods}

A SurveyMonkey ${ }^{\circledR}$ questionnaire was emailed to all postgraduate trainees in the East Midlands and Wessex deaneries. The survey was also disseminated via rota coordinators within surrounding NHS trusts. Data collection occurred between 1 and 20 July 2020.

\section{Results and discussion}

A total of 778 trainees completed the survey. Responses were equally split between both deaneries and represented a range of specialties (Fig 1). The majority of respondents were registrars (35\%), followed by foundation trainees $(25 \%)$, core trainees $(23 \%)$, general practice trainees ( $12 \%)$, trust grade/fellows (3\%) and 'others' $(2 \%)$.

On analysis of free-text data, additional skills gained were categorised, in order of decreasing frequency, into: clinical (diagnostics, escalation of care, general medical knowledge, invasive and non-invasive ventilation); challenging communication; remote consultation; infection control (donning and doffing of personal protective equipment); procedural (chest drain, arterial and central line insertion); leadership and management (rota planning, guideline and service development); education (e-learning design and delivery); and COVID-19 research.

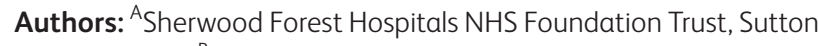
in Ashfield, UK; ${ }^{B}$ Portsmouth Hospitals NHS Trust, Portsmouth, UK; ' King's College Hospital NHS Foundation Trust, London, UK;

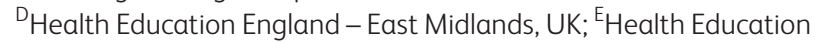
England - Wessex, UK

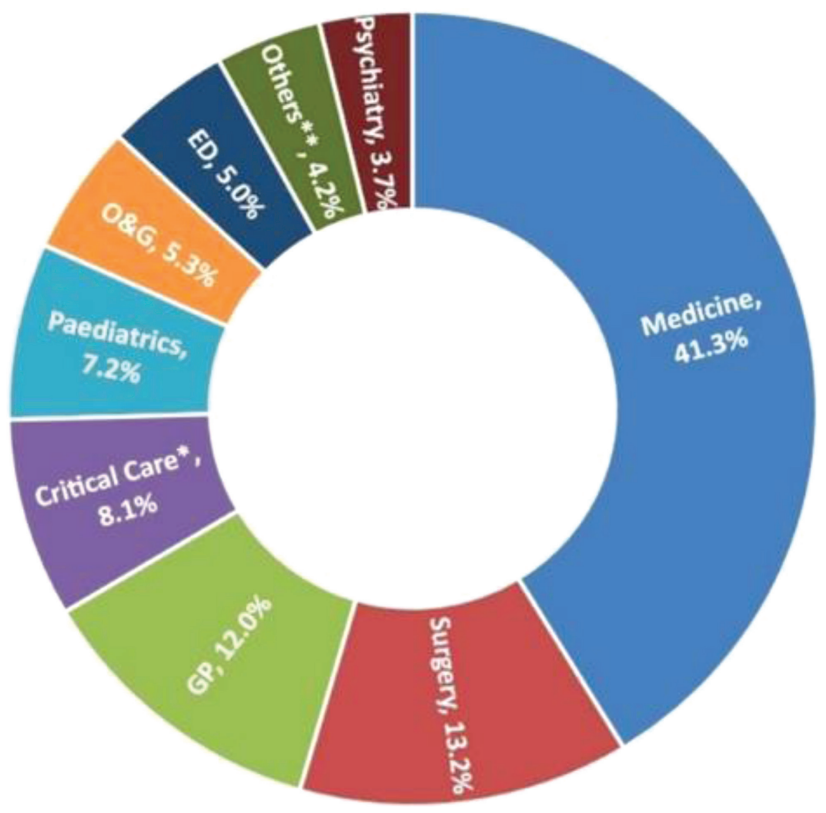

Fig 1. Clinical specialty of respondents. *Includes anaesthetics; **includes radiology, dentistry, genetics, pathology, and public health. ED = emergency department; GP = general practice; $O \& G$ = obstetrics and gynaecology.

Views on the usefulness of a COVID certificate were varied $(n=729)-72 \%$ positive, $17 \%$ negative and $11 \% \mathrm{mixed} /$ neutral. Most welcomed the proposal as a source of 'reward', 'confidence booster' and 'recognition' for their 'hard work', but also as a record of learning during the temporary 'pause in training'. However, it was perceived that a 'generic' document would lack competitive advantage and be superfluous to existing e-portfolios. Others cited concerns about additional bureaucratic workload and lack of formal endorsement for use during Annual Review of Competence Progression (ARCP). Some viewed the process as potentially discriminatory to those who were not formally redeployed, including 'shielding' colleagues, who may be perceived as less deserving. In view of these responses, it was apparent that self-certification should be voluntary and adaptable to suit diverse experiences, with minimal administrative burden. 


\section{WHS}

\section{COVID SELF-CERTIFICATION}

Name:

Professional registration number:

HEE region/Employer:

Area/department of redeployment:

Name of hospital where redeployed

Duration of redeployment:

Describe any changes to your role during the COVID-19 pandemic

Describe any clinical experience and knowledge gained relevant to your normal speciality and/or training programme during COVID-19.

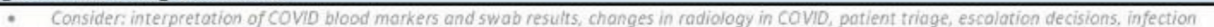
control/PPE, surgicol management of COVD patients. This could be cross-referenced to leaming objectives in your curriculum or syllabus

Describe any experience and knowledge gained in general medicine that is outside your usual scope of practice (i.e. palliative care, virtual clinics, rehabilitation, infection control, medicine prescribing)

- Consider genenal medical on calls for trainees or staff outside medicine?

Describe any experience and knowledge gained in a level 2 or 3 setting that is outside your usual scope of practice e.g. Critical Care or Medical High Dependency Unit

- Consider experiences in NIV, CPAP

- To avoid duplication, consider completing the co vid 'skills passport' if mare applicable to you

Describe any transferable skills and experience related to GMC's Generic Professional Capabilities https://www. gmc-uk.org/education/standards-guidance-and-curricula/standards-and-outcomes/generic-professional-capabilities-framework

- Damain 2: Professional vafues and behoviours

- Domain 2: Professional skills the proctical/cdinicaf skillts, communication, dealing with uncertainty

- Domain 3: Professional knowledge

- Domain 4: Capabulities in heolth promotion and flliness prevention

- Domain 5: Capabilties in leadership and team working

- Domain 6: Capabinties in patient safety and quality improvement

- Domain 7: Capabilaties in sofeguarding vulnerable groups

- Domain 8: Capabilities in education anduraining

- Domain 9: Copabilities in reszanch and scholarshn

Reflection could be documented here or indicate where in e-Portfolio reflection can be found.

- This section could also be used to document anyskifils/experiences that has not been included in any of the above sections

Please indicate $X$, if you have completed a reflection on your experience: Yes

Health Professional name

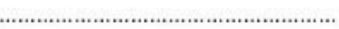

Role/Grade/Band

Health Professionale-signature

.........................................................

Date
No

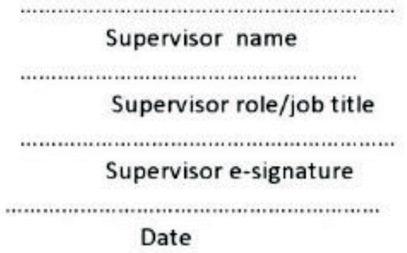

Date
Fig 2. The COVID self-certification template.
We would appreciate your feedback AFTER you have completed the self-certification process. Please click on this link https://www.surveymonkey.co.uk/r/TBN3SCM

COVID self-certification 21*July 2020 (Final V1)

Page 1

\section{Conclusion}

We have created a COVID self-certification template (Fig 2) in collaboration with postgraduate trainee representatives, the Faculty of Intensive Care Medicine and Health Education England. ${ }^{2}$
The template is suitable for use by any medical or non-medical healthcare professional. It provides an account of additional capabilities acquired during the pandemic, which may be used as evidence towards attaining curriculum competencies or professional revalidation. It may also facilitate workforce planning 
and future redeployment to areas of clinical need to ensure provision of safe, prompt and effective patient care. A follow-up survey $^{3}$ is currently underway to capture user feedback on the selfcertification process.

\section{Conflicts of interest}

None declared.

\section{References}

1 Joint Royal Colleges of Physicians Training Board (JRCPTB). ARCP decision aid for JRCPTB specialties during the COVID-19 pandemic
JRCPTB, 2020. www.jrcptb.org.uk/sites/default/files/JRCPTB \% 20ARCP \% 20decision \% 20aid \% 20for \% 20Covid \% 2019\% 20 pandemic.pdf [Accessed 09 September 2020].

2 Faculty of Intensive Care Medicine. FICM and HEE launch COVID Skills Passport. FICM, 2020. www.ficm.ac.uk/news-events-education/news/ficm-and-hee-launch-covid-skills-passport [Accessed 9 September 2020].

3 COVID self-certification post-completion survey. www. surveymonkey.co.uk/r/TBN3SCM [Accessed 2 December 2020]. 